\title{
Effect of nitrogen on intake and digestibility of a tropical grass grazed by Creole heifers
}

\author{
M. BOVAL ${ }^{1 *}$, P. CRUZ ${ }^{2}$, J. E. LEDET ${ }^{1}$, O. COPPRY ${ }^{1}$ AND H. ARCHIMEDE ${ }^{1}$ \\ ${ }^{1}$ Institut National de la Recherche Agronomique (INRA) Antilles-Guyane, Station de Zootechnie, \\ Domaine Duclos, 97170 Petit-Bourg, Guadeloupe (F.W.I.) \\ ${ }^{2}$ Institut National de la Recherche Agronomique (INRA) Toulouse, Station d'Agronomie, BP 27, \\ 31326 Castanet-Tolosan, France \\ (Revised MS received 20 June 2001)
}

\begin{abstract}
SUMMARY
So far, little has been done on the effect of nitrogen fertilizer on intake and digestibility in tropical grazing conditions. The effect of two nitrogen levels, 0 (NF) and $50 \mathrm{~kg} / \mathrm{ha}$ (F) for three successive grazing cycles, on organic matter intake (OMi) and digestibility (OMd) for Creole heifers $(208 \mathrm{~kg}$ live weight) was determined. Two groups of four tethered heifers, allowed $18 \mathrm{~kg}$ of dry matter daily, grazed individual NF or F circular areas at 28 re-growth days. The heifers were moved and watered daily. Sward characteristics (height, herbage mass, morphological and chemical composition and in situ tiller measurements), OMi, OMd and feeding behaviour (biting rate, bite size, intake rate, grazing and ruminating times) were evaluated simultaneously per circular area.

Under nitrogen fertilizer, the stems elongated rapidly (by 133\%) as did the leaves, but to a lesser extent (by $40 \%$ ). Consequently, canopy height, leaf and stem masses and crude protein content increased (respectively by $100 \%, 66 \%, 186 \%$ and $40 \%$ ), while total bulk density decreased (by $10.5 \%$ ). Throughout the successive grazing cycles, there was a particular effect on leaf number, both in the $\mathrm{F}$ and NF swards, whereas this parameter was not affected by the fertilizer level. Under fertilizer, OMi and OMd were both $9 \%$ greater than for the NF sward. The increase in herbage CP content partly explains the rise in $\mathrm{OMi}$, and there is evidence that rumen load has a determining effect on OMi. Leaf mass is the major factor accounting for the $9 \%$ increase in $\mathrm{OMd}$ under fertilizer regime. On the other hand, whatever the fertilizer regime, OMd was positively influenced by the greater leaf number throughout the grazing cycles. Biting rate, bite size and intake rate were determined respectively by sward height, CP content and stem fraction, whereas grazing and ruminating times were partially influenced by sward characteristics.
\end{abstract}

\section{INTRODUCTION}

Nitrogen fertilizer is commonly used to increase animal production at pasture. In tropical pastures, the live weight (LW) gain reported varies between $1 \cdot 3$ and $4.7 \mathrm{~kg} \mathrm{LW} /$ ha per year per additional $\mathrm{kg}$ of nitrogen added per hectare (Mears \& Humphreys 1974; Jones 1990; Humphreys 1991). In most studies the greater herbage mass or crude protein content induced by the addition of nitrogen fertilizer are the main parameters put forward to explain the rise in animal production. However, few data in the tropics allow a precise explanation of the mechanism by which nitrogen fertilizer affects the intake of grazing animals. Nevertheless, such knowledge would be

* To whom all correspondence should be addressed. Email: boval@antilles.inra.fr helpful for establishing a suitable fertilizer supply level, and also for developing alternative means to the addition of nitrogen fertilizer to generate greater intake at pasture.

Using equal herbage allowances, the effect of fertilizing an indigenous tropical sward based on Dichanthium spp., grazed by Creole heifers, on intake and on digestibility was investigated. The primary aim was to estimate the gain in intake and digestibility under fertilization, as these two parameters determine LW gain. A second objective was to determine any additional parameters involved in the improvement of gain.

\section{MATERIALS AND METHODS}

The experiment was carried out at the experimental station of the National Institute for Agricultural 
Research (INRA) in the French West Indies, Guadeloupe $\left(16^{\circ} 16^{\prime} \mathrm{N}, \quad 61^{\circ} 30^{\prime} \mathrm{W}\right)$. Average temperatures ranged from $21-25^{\circ} \mathrm{C}$ to $27-31{ }^{\circ} \mathrm{C}$. The mean rainfall on the experimental site is $1000 \mathrm{~mm}$ a year.

\section{Experimental design}

Two levels of nitrogen fertilizer ( 0 and $50 \mathrm{~kg} / \mathrm{ha}$ ) were applied to two plots of a sward based on Dichanthium spp. after 28 days re-growth. The plots were grazed by two groups of 4 tethered Creole heifers (12 months old and $208 \mathrm{~kg} \mathrm{LW}$ ), during three successive grazing cycles, according to a $2 \times 2$ Latin Square design. Each grazing cycle comprised two 4-day measurement periods, each following an 11-day adaptation period. The measurements were taken every 15 days. During the same grazing cycle, the heifers grazed alternately NF or F sward. They had a daily allowance of $18 \mathrm{~kg}$ of dry matter (DM).

\section{Sward and animal management}

Two plots were each divided into 28 subplots in early February. The subplots were all fertilized at the beginning of the experiment with $187 \mathrm{~kg} / \mathrm{ha}$ of a 27-9-18 N-P-K fertilizer, in order to respect the annual management of this natural grassland. Every day from 24 February to 24 March, two subplots per plot were mown daily at ground level with a powered lawnmower equipped with a collection tray. After mowing, one of the mown subplots was fertilized $(\mathrm{F})$ with $50 \mathrm{~kg}$ nitrogen per hectare as ammonium nitrate $\left(\begin{array}{ll}\mathrm{NH}_{4} & \mathrm{NO}_{3}\end{array}\right)$ whereas the second was not (NF). In order to avoid limiting water conditions, a positive water balance was maintained by irrigating all the plots, in accordance with the evapo-transpiration estimated from data collected by an adjacent automatic meteorological station (CIMEL). On 24 March, the last two subplots $\mathrm{F}$ and NF were mown which subsequently, together with those mown first, had their 28 day re-growth grazed for the first time by the heifers for $24 \mathrm{~h}$. The first grazing cycle then began. Afterwards the subplots were grazed successively every 28 days. The other grazing cycles lasted 28 days each.

The two groups of 4 heifers grazed two subplots NF or F daily. Each of the eight heifers had a defined circular area of herbage to graze, determined by the chain length and the herbage mass, measured previously, and taking into account the heifer neck length. They were moved daily to fresh areas at $09.00 \mathrm{~h}$ and were watered individually at $13.00 \mathrm{~h}$. Every 15 days, at the end of each period of measurement, the heifers were weighed at $09.00 \mathrm{~h}$ and were then treated against ticks by spraying. An anthelminthic treatment was applied once a month.

\section{Sward characterization}

The sward which the heifers were allowed to graze was characterized by physical and chemical parameters. Swards were measured for each of the eight circular areas of herbage intended for daily grazing by the heifers, for 2 days per measurement period. Sward height was measured with a rising-plate meter (Michell 1982) at 10 sites per circular area. Herbage mass was estimated at the same sites, by cutting the herbage under the plate over an area of $0.03 \mathrm{~m}^{2}$, at ground level with hand-held electric clippers. Each of the 10 herbage samples cut was weighed fresh, and all of them were then pooled per circular area. Two subsamples of $300 \mathrm{~g}$ were kept, the first to determine dry matter (DM) and chemical composition, the second to determine morphological composition: stems, leaves and debris (including senescent and dead material) were sorted manually prior to drying.

The structure of in situ tillers was described before grazing. Ten random tillers per circular area $(0 \cdot 14$ to 0.28 tillers $/ \mathrm{m}^{2}$ ) were identified with a coloured ring, and for each one, extended length, stem length and leaf length were measured with a sliding ruler. The cumulated leaf length per tiller was calculated by adding all the leaf lengths from the same tiller. Total herbage bulk density $\left(\mathrm{kg} \mathrm{OM} / \mathrm{m}^{3}\right)$ before grazing, was calculated by dividing the total herbage mass $\left(\mathrm{kg} \mathrm{OM} / \mathrm{m}^{2}\right)$ by the mean extended tiller length $(\mathrm{m})$. Stem and leaf bulk densities were also calculated by dividing the stem and leaf masses by the mean extended tiller length.

\section{Determining $O M$ intake and $O M$ digestibility}

Organic matter intake per day (OMi, kg OM/day) was determined from total faecal OM output and OM digestibility (Streeter 1969): OMi = faecal OM output /(1-OM digestibility).

Faecal OM output per day (kg OM/day) was measured by manually collecting all faeces excreted by each heifer on the individual circular areas, over the 4-day measurement period. Faeces collection was facilitated by the high faecal DM content $(184 \mathrm{~g} / \mathrm{kg}$ faeces on average) and was carried out twice daily $(07.00 \mathrm{~h}$ and $17.00 \mathrm{~h})$ to reduce non-collection of faeces due to trampling. The amount of uncollected faeces was estimated to be $2 \%$ of the total amount excreted (Boval et al. 1996b). For each heifer, the entire amount of collected faeces over the 4-day measurement period was weighed and calculated per day. The faeces were then mixed and homogenized and a subsample of $500 \mathrm{~g}$ was taken to determine chemical composition.

$\mathrm{OM}$ digestibility (OMd) was estimated for each heifer from the crude protein content (CP, g/ $\mathrm{kg} \mathrm{OM}$ ) of the faecal subsample taken, according to a local 
equation established by Boval et al. (1996a) with Creole steers fed on Dichanthium spp. herbage:

$\mathrm{OMd}=0.983-4 \cdot 002 / \mathrm{CP}$

$$
\left(R^{2}=0.84 ; \text { S.E. }=2.5310^{-2} ; \text { D.F. }=37\right) .
$$

Digestible OMi (dOMi, g OM/kg LW LW $^{0.75}$ per day) was calculated by multiplying $\mathrm{OMi}$, expressed by metabolic LW kg (g OM/kg LW ${ }^{0.75}$ per day), by OMd. Non digestible OMi (ndOMi, g OM $/ \mathrm{kg} \mathrm{LW}^{0.75}$ per day) is equal to faecal output expressed per $\mathrm{kg}$ of metabolic LW.

\section{Determining ingestive behavioural parameters}

The feeding behavioural parameters were determined by visually observing the heifers for $24 \mathrm{~h}$ twice per measurement period. The observers recorded the current activity of each heifer every $10 \mathrm{~min}$ : grazing (head down, searching for or gripping herbage), ruminating or idling. When the activity was grazing, the observer counted the number of bites (by listening to the noise of the herbage pulled out) taken for $1 \mathrm{~min}$, to determine the biting rate (BR, bites/min). At night, heifers were observed with a flashlight. Grazing, ruminating and idling times (GT, RT and IT respectively) were calculated by multiplying the times of each activity recorded every $10 \mathrm{~min}$, by 10 . Bite size (BS, mg OM) was calculated by dividing OMi by the total number of bites $(=\mathrm{BR} \times \mathrm{GT})$. Intake rate (IR, $\mathrm{g}$ $\mathrm{OM} / \mathrm{min}$ ) was calculated by multiplying biting rate by bite size.

\section{Determining chemical composition}

Dry matter (DM) contents of both herbage and faeces samples were determined by drying at constant weight at $60{ }^{\circ} \mathrm{C}$ in a forced-draught oven over $48 \mathrm{~h}$. The samples were then ground $(0 \cdot 75 \mathrm{~mm})$ prior to chemical analysis. The $\mathrm{OM}$ content was measured after a $10 \mathrm{~h}$ pyrolysis at $550{ }^{\circ} \mathrm{C}$. Neutral detergent fibre (NDF), acid detergent fibre (ADF) and acid detergent lignin (ADL) were estimated following the methods of Van Soest et al. (1991). Nitrogen concentration was determined by the Kjeldahl method. Crude protein content was calculated by multiplying the nitrogen content by $6 \cdot 25$.

\section{Statistical analysis}

Data were analysed according to a Latin Square design, with the GLM (General Linear Model) procedure of SAS (1989). Animal data and in situ tiller measurements were analysed according to the following model:

$$
\mathrm{Y}_{\mathrm{ijk}}=\mathrm{m}+\mathrm{F}_{\mathrm{i}}+\mathrm{G}_{\mathrm{j}}+\mathrm{H}_{\mathrm{k}}+(\mathrm{FG})_{\mathrm{ij}}+\mathrm{P}_{1}\left(\mathrm{G}_{\mathrm{j}}\right)+\mathrm{e}_{\mathrm{ijk} \mathrm{k}}
$$

where $m$ is the mean, $F_{i}$ is the fertilization effect $(i=$ $1,2), G_{j}$ is the grazing cycle effect $(j=1,2,3), H_{k}$ is the heifer effect $(\mathrm{k}=1$ to 8$),(\mathrm{FG})_{\mathrm{ij}}$ is the fertilization $\times$ grazing cycle interaction effect, $P_{1}\left(G_{j}\right)$ is the period effect $(1=1,2)$ within each grazing cycle and $\mathrm{e}_{\mathrm{ijkl}}$ is the residual term. The other herbage data (sward height, herbage mass and morphological parameters) were analysed according to the same model, without the heifer effect.

The relationships between all the measured parameters were first shown by calculating correlation coefficients by the COR procedure of SAS (1989). Regressions were then calculated by the REG procedure using the MAXR (Maximum $R^{2}$ Improvement) method. This method tests each independent parameter with all the others, until the best $n$-parameter model ( $n$ fixed by user) is obtained, producing the highest $R^{2}$. The dependent variables were corrected for the heifer effect. The residual distribution was analysed.

\section{RESULTS}

\section{Effect of nitrogen fertilizer and grazing cycles on sward characteristics}

Under nitrogen fertilizer, the herbage mass increased by $1.6 \mathrm{t} \mathrm{DM} /$ ha, and the sward height was nearly twice that of the NF sward (Table 1). Both stem and leaf masses increased with nitrogen fertilizer, but not to the same extent (186 v. 66\% respectively), and consequently the leaf/stem ratio decreased. Debris mass remained the same whatever the fertilizer level. The total and leaf bulk density values both decreased with fertilizer use (by $11 \%$ and $14 \%$ respectively), whereas the bulk density of the stems rose by $57 \%$. The fertilized tillers grew much longer than the unfertilized ones, with the extended tiller length increasing by $67 \%$. The stem and leaf lengths both increased but not to the same extent (by $133 \%$ and $39 \%$ respectively), whereas the leaf number per tiller remained the same for the $\mathrm{F}$ and the NF swards. The main chemical parameter modified by nitrogen fertilizer was the herbage CP content $(40 \%$ higher in the F sward).

Throughout the successive grazing cycles on the NF sward, the leaf mass, leaf length and also the number of leaves, increased respectively, by $113 \%$, $16 \%$ and $21 \%$ (Table 1 ). Leaf bulk density rose by $133 \%$ and consequently total bulk density increased by $52 \%$. In the $\mathrm{F}$ sward, the grazing cycles had a significant effect on all the herbage characteristics, not only on the leaves as was the case for the NF sward. Canopy height as well as total, stem and leaf masses, increased sharply between the first and the third grazing cycle. All the morphological parameters were also significantly greater at the third grazing cycle than at the first.

\section{Effect of nitrogen fertilizer and grazing cycles on intake, digestibility and ingestive behaviour}

On the F sward, the heifers ingested 9\% more OM and the herbage ingested was better digested by 6 
Table 1. Characteristics of unfertilized $(N F)$ and fertilized $(F)$ tropical Dichanthium spp. based swards, each grazed by tethered Creole heifers, during three grazing cycles (NF1, NF2, NF3 and F1, F2, F3)

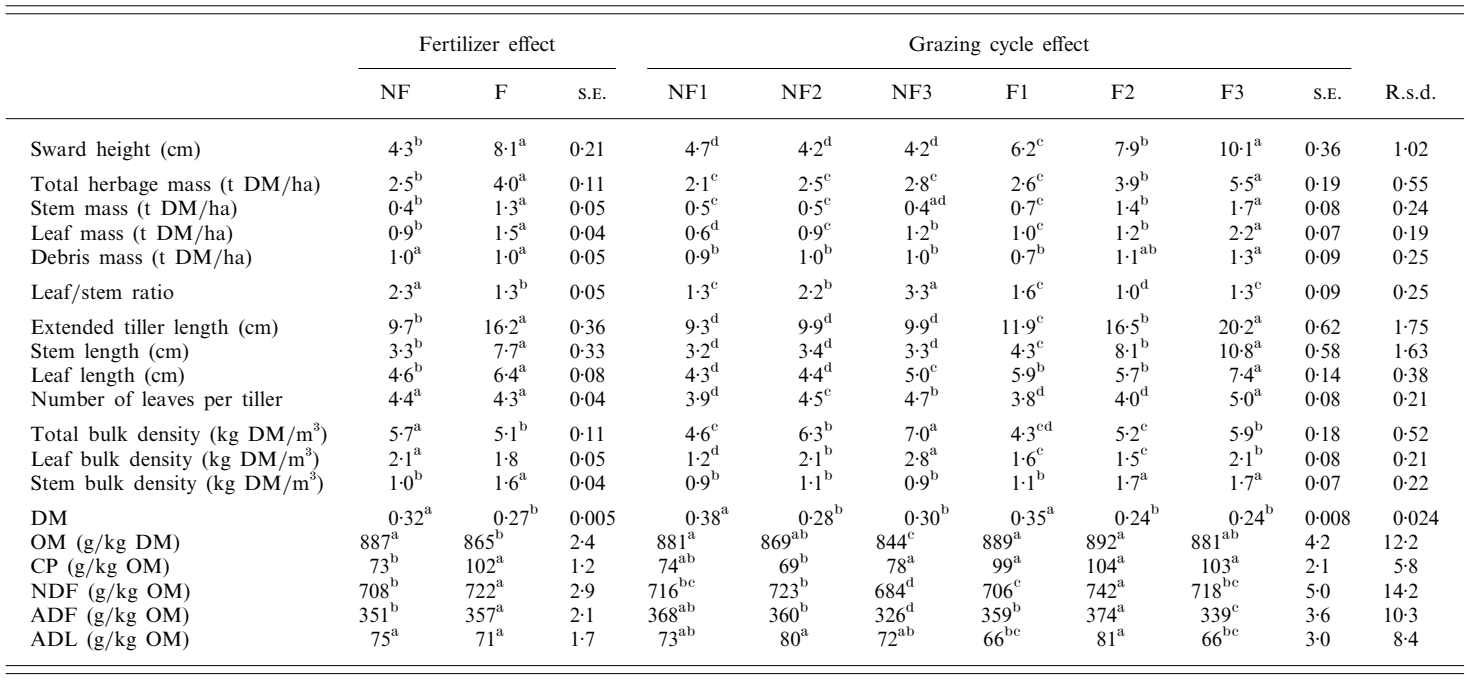

Values with different superscripts within fertilizer levels or grazing cycles are significantly different $(P<0 \cdot 05)$.

R.s.d.: Root Mean Squared Error; S.E.: standard error.

Table 2. Herbage allowances, OM intake, OM digestibility and ingestive behaviour parameters (Biting rate, Bite size, Intake rate, Grazing, Ruminating and Idling times) for tethered Creole heifers grazing unfertilized (NF) or fertilized $(F)$ tropical Dichanthium spp. based swards, during three grazing cycles $(N F, N F 2, N F 3$ and F1, $F 2, F 3)$

\begin{tabular}{|c|c|c|c|c|c|c|c|c|c|c|c|}
\hline & \multicolumn{3}{|c|}{ Fertilizer effect } & \multicolumn{7}{|c|}{ Grazing cycle effect } & \multirow[b]{2}{*}{ R.s.d. } \\
\hline & $\mathrm{NF}$ & $\mathrm{F}$ & S.E. & NF1 & NF2 & NF3 & $\mathrm{F} 1$ & F2 & F3 & S.E. & \\
\hline Allowance (g OM/kg LW ${ }^{0} 75$ per day) & $342^{\mathrm{a}}$ & $351^{\mathrm{a}}$ & $10 \cdot 4$ & $297^{\mathrm{c}}$ & $343^{b}$ & $386^{\mathrm{a}}$ & $287^{\mathrm{c}}$ & $389^{\mathrm{a}}$ & $377^{\mathrm{ab}}$ & $18 \cdot 1$ & 3.5 \\
\hline OM intake $\left(\mathrm{g} / \mathrm{kg} \mathrm{LW} \mathrm{W}^{0^{075}}\right.$ per day) & $76 \cdot 6^{\mathrm{b}}$ & $83 \cdot 6^{\mathrm{a}}$ & 1.53 & $75 \cdot 1^{\mathrm{b}}$ & $77 \cdot 1^{\mathrm{b}}$ & $77 \cdot 6^{\mathrm{b}}$ & $81 \cdot 7^{\mathrm{ab}}$ & $89 \cdot 1^{\mathrm{a}}$ & $80 \cdot 1^{\mathrm{b}}$ & $2 \cdot 65$ & $7 \cdot 50$ \\
\hline OM digestibility & $0.64^{\mathrm{b}}$ & $0.70^{\mathrm{a}}$ & 0.002 & $0.62^{\mathrm{f}}$ & $0.63^{\mathrm{e}}$ & $0.67^{\mathrm{d}}$ & $0.68^{\mathrm{c}}$ & $0 \cdot 70^{\mathrm{b}}$ & $0.73^{\mathrm{a}}$ & 0.004 & $0 \cdot 012$ \\
\hline $\begin{array}{l}\text { dOMi }\left(\mathrm{g} / \mathrm{kg} \mathrm{LW} \mathrm{LW}^{0 \cdot 75} \text { per day) }\right. \\
\text { ndOMi }\left(\mathrm{g} / \mathrm{kg} \mathrm{LW} \mathrm{LW}^{0.75} \text { per day }\right)\end{array}$ & $\begin{array}{l}49 \cdot 3^{\mathrm{b}} \\
27 \cdot 6^{\mathrm{a}}\end{array}$ & $\begin{array}{l}59 \cdot 5^{\mathrm{a}} \\
24 \cdot 9^{\mathrm{b}}\end{array}$ & $\begin{array}{l}1 \cdot 11 \\
0 \cdot 48\end{array}$ & $\begin{array}{l}46 \cdot 6^{\mathrm{bc}} \\
28 \cdot 6^{\mathrm{a}}\end{array}$ & $\begin{array}{l}48 \cdot 8^{\mathrm{bc}} \\
28 \cdot 3^{\mathrm{a}}\end{array}$ & $\begin{array}{l}51 \cdot 5^{\mathrm{b}} \\
26 \cdot 1^{\mathrm{ab}}\end{array}$ & $\begin{array}{l}55 \cdot 8^{\mathrm{ab}} \\
25 \cdot 9^{\mathrm{b}}\end{array}$ & $\begin{array}{l}62 \cdot 0^{\mathrm{a}} \\
27 \cdot \mathrm{a}^{\mathrm{ab}}\end{array}$ & $\begin{array}{l}58 \cdot 3^{\mathrm{a}} \\
21 \cdot 9^{\mathrm{c}}\end{array}$ & $\begin{array}{l}1 \cdot 93 \\
0.84\end{array}$ & $\begin{array}{l}5 \cdot 46 \\
2 \cdot 37\end{array}$ \\
\hline $\begin{array}{l}\text { Biting rate }(\mathrm{bites} / \mathrm{min}) \\
\text { Bite size }(\mathrm{mg} \mathrm{OM}) \\
\text { Intake rate }(\mathrm{g} \mathrm{OM} / \mathrm{min})\end{array}$ & $\begin{array}{r}54^{\mathrm{a}} \\
186^{\mathrm{b}} \\
10^{\mathrm{b}}\end{array}$ & $\begin{array}{r}50^{\mathrm{b}} \\
267^{\mathrm{a}} \\
13^{\mathrm{a}}\end{array}$ & $\begin{array}{l}0 \cdot 6 \\
6 \cdot 2 \\
0 \cdot 3\end{array}$ & $\begin{array}{l}54^{\mathrm{b}} \\
205^{\mathrm{c}} \\
10 \cdot 9^{\mathrm{b}}\end{array}$ & $\begin{array}{c}55^{\mathrm{b}} \\
172^{\mathrm{d}} \\
9 \cdot 2^{\mathrm{c}}\end{array}$ & $\begin{array}{c}58^{\mathrm{a}} \\
181^{\mathrm{cd}} \\
10 \cdot 0^{\mathrm{be}}\end{array}$ & $\begin{array}{l}53^{\mathrm{bc}} \\
247^{\mathrm{b}} \\
12 \cdot 8^{\mathrm{a}}\end{array}$ & $\begin{array}{l}51^{\mathrm{cd}} \\
280^{\mathrm{a}} \\
13 \cdot 8^{\mathrm{a}}\end{array}$ & $\begin{array}{c}49^{\mathrm{d}} \\
276^{\mathrm{ab}} \\
\quad 12 \cdot 8^{\mathrm{a}}\end{array}$ & $\begin{array}{r}1 \cdot 1 \\
10 \cdot 7 \\
0 \cdot 5\end{array}$ & $\begin{array}{r}3 \cdot 2 \\
30 \cdot 4 \\
1 \cdot 5\end{array}$ \\
\hline $\begin{array}{l}\text { Grazing time }(\mathrm{min}) \\
\text { Ruminating time }(\mathrm{min}) \\
\text { Idling time (min) }\end{array}$ & $\begin{array}{l}431^{\mathrm{a}} \\
129^{\mathrm{a}} \\
213^{\mathrm{b}}\end{array}$ & $\begin{array}{l}362^{\mathrm{b}} \\
179^{\mathrm{a}} \\
233^{\mathrm{a}}\end{array}$ & $\begin{array}{l}6 \cdot 0 \\
5 \cdot 7 \\
6 \cdot 8\end{array}$ & $\begin{array}{l}378^{\mathrm{b}} \\
189^{\mathrm{b}} \\
233^{\mathrm{a}}\end{array}$ & $\begin{array}{l}463^{\mathrm{a}} \\
101^{\mathrm{d}} \\
197^{\mathrm{b}}\end{array}$ & $\begin{array}{r}452^{\mathrm{a}} \\
98^{\mathrm{d}} \\
208^{\mathrm{ab}}\end{array}$ & $\begin{array}{l}351^{\mathrm{b}} \\
219^{\mathrm{a}} \\
232^{\mathrm{a}}\end{array}$ & $\begin{array}{l}367^{\mathrm{b}} \\
160^{\mathrm{c}} \\
233^{\mathrm{a}}\end{array}$ & $\begin{array}{l}368^{\mathrm{b}} \\
157^{\mathrm{c}} \\
233^{\mathrm{a}}\end{array}$ & $\begin{array}{r}10 \cdot 4 \\
9.8 \\
11 \cdot 8\end{array}$ & $\begin{array}{l}29 \cdot 5 \\
27 \cdot 8 \\
33 \cdot 4\end{array}$ \\
\hline
\end{tabular}

Values with different superscripts within fertilizer levels or grazing cycles are significantly different $(P<0 \cdot 05)$.

dOMi, digestible OM intake; ndOMi, non digestible OM intake; R.s.d., Root Mean Squared Error; s.E., standard error.

digestibility units than on the NF sward (Table 2). On the $\mathrm{F}$ sward the heifers grazed with a lower biting rate and for a shorter time than on the NF sward. Bite size and intake rate were both higher on the F sward.

From the first to the last grazing cycle, with both the NF and the F swards, digestibility increased by 5 digestibility units whereas intake did not vary (Table 2). On the F sward, the biting rate decreased whereas the grazing time did not vary between the grazing cycles. On the NF sward, both the biting rate and the grazing time increased (Table 2) from the first to the third grazing cycle.

Analysis of determining parameters for intake, digestibility and ingestive behaviour

Considering the entire sward data, OMi was mostly 
Table 3 a. Correlation coefficients between $O M$ intake $(O M i), O M$ digestibility $(O M d)$, Biting rate $(B R$, bites/min), Bite size (BS, mg OM), Intake rate ( $\mathrm{g} O M /$ min), Grazing time $(G T$, min) and Ruminating time $(R T$, min) and sward characteristics, whatever the fertilization level, for tethered Creole heifers grazing tropical Dichanthium spp. based swards

\begin{tabular}{|c|c|c|c|c|c|c|c|}
\hline & OMi & OMd & BR & BS & IR & GT & RT \\
\hline Sward height $(\mathrm{cm})$ & $0 \cdot 24^{\mathrm{ns}}$ & $0 \cdot 70^{* * *}$ & $-0 \cdot 71 * * *$ & $0 \cdot 70^{* * *}$ & $0 \cdot 39 * *$ & $-0 \cdot 30^{*}$ & $0 \cdot 26^{\mathrm{ns}}$ \\
\hline $\begin{array}{l}\text { Total mass (t DM/ha) } \\
\text { Stem mass (t DM/ha) } \\
\text { Leaf mass (t DM/ha) } \\
\text { Debris mass (t DM/ha) }\end{array}$ & $\begin{array}{l}0 \cdot 29^{*} \\
0 \cdot 33^{*} \\
0 \cdot 21^{\mathrm{ns}} \\
0 \cdot 23^{\mathrm{ns}}\end{array}$ & $\begin{array}{l}0 \cdot 73^{* * *} \\
0 \cdot 74^{* * *} \\
0 \cdot 77^{* * *} \\
0 \cdot 24^{\text {ns }}\end{array}$ & $\begin{array}{l}-0 \cdot 60^{* * *} \\
-0 \cdot 61 * * * \\
-0 \cdot 51 * * * \\
-0 \cdot 48^{* * *}\end{array}$ & $\begin{array}{l}0 \cdot 61^{* * *} \\
0 \cdot 72^{* * *} \\
0 \cdot 51^{* * *} \\
0 \cdot 27^{*}\end{array}$ & $\begin{array}{l}0 \cdot 33^{* *} \\
0 \cdot 46^{* * *} \\
0 \cdot 27^{\mathrm{ns}} \\
0 \cdot 01^{\mathrm{ns}}\end{array}$ & $\begin{array}{r}-0 \cdot 18^{\mathrm{ns}} \\
-0 \cdot 31^{*} \\
-0 \cdot 16^{\mathrm{ns}} \\
0 \cdot 17^{\mathrm{ns}}\end{array}$ & $\begin{array}{r}0 \cdot 05^{\mathrm{ns}} \\
0 \cdot 11^{\mathrm{ns}} \\
-0 \cdot 03^{\mathrm{ns}} \\
0 \cdot 14^{\mathrm{ns}}\end{array}$ \\
\hline $\begin{array}{l}\text { Leaf/stem ratio } \\
\text { Extended tiller length }(\mathrm{cm}) \\
\text { Stem length }(\mathrm{cm}) \\
\text { Mean leaf length }(\mathrm{cm}) \\
\text { Number of leaf per tiller }\end{array}$ & $\begin{array}{c}-0 \cdot 16^{\text {ns }} \\
0 \cdot 26^{\text {ns }} \\
0 \cdot 24^{\text {ns }} \\
0 \cdot 28^{\text {ns }} \\
0 \cdot 04^{\text {ns }}\end{array}$ & $\begin{array}{r}-0 \cdot 25^{\text {ns }} \\
0 \cdot 70^{* * *} \\
0 \cdot 65^{* * *} \\
0.75^{* * *} \\
0 \cdot 40^{* *}\end{array}$ & $\begin{array}{l}0 \cdot 40^{* *} \\
-0.66^{* * *} \\
-0 \cdot 65^{* * *} \\
-0.59^{* * *} \\
-0 \cdot 27^{\mathrm{ns}}\end{array}$ & $\begin{array}{r}-0.58^{* * *} \\
0.69^{* * *} \\
0 \cdot 68^{* * *} \\
0 \cdot 63^{* * *} \\
0.04^{\mathrm{ns}}\end{array}$ & $\begin{array}{c}-0 \cdot 48^{* * *} \\
0 \cdot 40^{* *} \\
0 \cdot 39^{* *} \\
0 \cdot 38^{* *} \\
-0 \cdot 15^{\mathrm{ns}}\end{array}$ & $\begin{array}{c}0 \cdot 48^{* * *} \\
-0 \cdot 28^{*} \\
-0 \cdot 28^{*} \\
-0 \cdot 26^{\mathrm{ns}} \\
0 \cdot 24^{\mathrm{ns}}\end{array}$ & $\begin{array}{c}-0 \cdot 40^{* *} \\
0 \cdot 14^{\text {ns }} \\
0 \cdot 07^{\text {ns }} \\
0 \cdot 31^{*} \\
-0 \cdot 33^{*}\end{array}$ \\
\hline $\begin{array}{l}\text { Total bulk density }\left(\mathrm{kg} \mathrm{DM} / \mathrm{m}^{3}\right) \\
\text { Leaf bulk density }\left(\mathrm{kg} \mathrm{DM} / \mathrm{m}^{3}\right) \\
\text { Stem bulk density }\left(\mathrm{kg} \mathrm{DM} / \mathrm{m}^{3}\right)\end{array}$ & $\begin{array}{l}0 \cdot 15^{\text {ns }} \\
0 \cdot 08^{\text {ns }} \\
0 \cdot 37^{* *}\end{array}$ & $\begin{array}{l}-0 \cdot 04^{\mathrm{ns}} \\
0 \cdot 15^{\mathrm{ns}} \\
0 \cdot 64^{* * *}\end{array}$ & $\begin{array}{c}0 \cdot 41^{* *} \\
0 \cdot 33^{*} \\
-0 \cdot 28^{\text {ns }}\end{array}$ & $\begin{array}{l}-0 \cdot 35^{*} \\
-0 \cdot 27^{\mathrm{ns}} \\
0 \cdot 61^{* * *}\end{array}$ & $\begin{array}{l}-0 \cdot 22^{\mathrm{ns}} \\
-0 \cdot 17^{\mathrm{ns}} \\
0 \cdot 54^{* * *}\end{array}$ & $\begin{array}{c}0 \cdot 44^{* *} \\
0 \cdot 33^{*} \\
-0 \cdot 30^{*}\end{array}$ & $\begin{array}{l}-0.53^{* * *} \\
-0.51^{* * *} \\
-0.04^{\mathrm{ns}}\end{array}$ \\
\hline $\begin{array}{l}\mathrm{CP}(\mathrm{g} / \mathrm{kg} \mathrm{OM}) \\
\mathrm{NDF}(\mathrm{g} / \mathrm{kg} \mathrm{OM}) \\
\text { ADF }(\mathrm{g} / \mathrm{kg} \mathrm{OM}) \\
\text { ADL }(\mathrm{g} / \mathrm{kg} \mathrm{OM})\end{array}$ & $\begin{array}{r}0 \cdot 43^{* *} \\
-0 \cdot 05^{\mathrm{ns}} \\
-0 \cdot 01^{\mathrm{ns}} \\
0 \cdot 18^{\mathrm{ns}}\end{array}$ & $\begin{aligned} & 0 \cdot 75^{* * *} \\
& 0 \cdot 17^{\mathrm{ns}} \\
- & 0 \cdot 21^{\mathrm{ns}} \\
- & 0 \cdot 29^{*}\end{aligned}$ & $\begin{array}{l}-0 \cdot 25^{\mathrm{ns}} \\
-0 \cdot 60^{* * *} \\
-0 \cdot 32^{*} \\
0 \cdot 05^{\mathrm{ns}}\end{array}$ & $\begin{array}{l}0 \cdot 79^{* * *} \\
0 \cdot 29^{*} \\
0 \cdot 12^{\text {ns }} \\
-0 \cdot 21^{\text {ns }}\end{array}$ & $\begin{array}{l}0 \cdot 77^{* * *} \\
0 \cdot 02^{\mathrm{ns}} \\
-0 \cdot 01^{\mathrm{ns}} \\
-0 \cdot 23^{\mathrm{ns}}\end{array}$ & $\begin{array}{l}-0 \cdot 61^{* * *} \\
-0 \cdot 10^{\mathrm{ns}} \\
-0 \cdot 03^{\mathrm{ns}} \\
0 \cdot 43^{* *}\end{array}$ & $\begin{array}{l}0 \cdot 03^{\text {ns }} \\
0 \cdot 20^{\text {ns }} \\
0 \cdot 45^{* *} \\
0 \cdot 05^{\text {ns }}\end{array}$ \\
\hline
\end{tabular}

ns, non significant; *,**,***: significantly correlated $(P<0 \cdot 01,0 \cdot 001,0 \cdot 0001$ respectively).

Table $3 b$. Correlation coefficients between $O M$ intake $\left(O M i, g / \mathrm{kL} \mathrm{LW}^{0 \cdot 75}\right)$, OM digestibility $(O M d)$, digestible $O M$ intake (dOMi, $\left.\mathrm{g} / \mathrm{kg} \mathrm{L} W^{0 \cdot 75}\right)$, non digestible OM intake (ndOMi, $\left.\mathrm{g} / \mathrm{kg} \mathrm{LW} W^{0 \cdot 75}\right)$, Biting rate $(B R$, bites $/ \mathrm{min})$, Bite size $(B S, m g O M)$, Intake rate $(g O M / m i n)$, Grazing time $(G T$, min) and Ruminating time (RT, min) for tethered Creole heifers grazing tropical Dichanthium spp. based swards, whatever the fertilization level

\begin{tabular}{|c|c|c|c|c|c|c|c|c|c|}
\hline & OMi & OMd & $\mathrm{dOMi}$ & ndOMi & BR & BS & IR & GT & RT \\
\hline OMd & $0 \cdot 21^{\mathrm{ns}}$ & & & & & & & & \\
\hline $\mathrm{dOMi}$ & $0.92 * * *$ & $0 \cdot 58 * * *$ & & & & & & & \\
\hline ndOMi & $0 \cdot 61 * *$ & $-0 \cdot 64 * * *$ & $0 \cdot 24^{\mathrm{ns}}$ & & & & & & \\
\hline BR & $0 \cdot 19^{\text {ns }}$ & $-0 \cdot 48^{* * *}$ & $-0.04^{\mathrm{ns}}$ & $0 \cdot 54 * * *$ & & & & & \\
\hline $\mathrm{BS}$ & $0 \cdot 52 * * *$ & $0.67 * * *$ & $0.71^{* * *}$ & $-0 \cdot 14^{\mathrm{ns}}$ & $-0 \cdot 49^{* * *}$ & & & & \\
\hline IR & $0 \cdot 70^{* * *}$ & $0 \cdot 48^{* * *}$ & $0 \cdot 79 * * *$ & $0 \cdot 14^{\text {ns }}$ & $-0.03^{\mathrm{ns}}$ & $0 \cdot 88^{* * *}$ & & & \\
\hline GT & $-0 \cdot 15^{\mathrm{ns}}$ & $-0 \cdot 30^{*}$ & $-0 \cdot 25^{\mathrm{ns}}$ & $0 \cdot 14^{\mathrm{ns}}$ & $0 \cdot 11^{\mathrm{ns}}$ & $-0.67 * * *$ & $-0.73^{* * *}$ & & \\
\hline RT & $0 \cdot 15^{\mathrm{ns}}$ & $0.02^{\mathrm{ns}}$ & $0 \cdot 14^{\mathrm{ns}}$ & $0 \cdot 07^{\mathrm{ns}}$ & $-0.32 *$ & $0 \cdot 19^{\mathrm{ns}}$ & $0 \cdot 09^{\mathrm{ns}}$ & $-0 \cdot 12^{\mathrm{ns}}$ & \\
\hline
\end{tabular}

ns, non significant; $*{ }^{* *}, * * *$ : significantly correlated $(P<0 \cdot 01,0 \cdot 001,0 \cdot 0001$ respectively).

correlated with the herbage $\mathrm{CP}$ content and stem bulk density (Table $3 a$ ). OMi was not correlated with OMd, even if these two variables are linked by calculation (Table $3 b$ ), but was correlated to dOMi and ndOMi. The dOMi increased with OMi on F or $\mathrm{NF}$ sward with a slope of $0 \cdot 75$, whereas ndOMi increased slowly, keeping close to an average value of $26 \cdot 3 \mathrm{~g} \mathrm{OM} / \mathrm{kg} \mathrm{LW}^{0.75}$, whatever the F or NF treatment (Fig. 1). Intake was also correlated with bite size and intake rate (Table $3 b$ ) but these correlations are also arguable, as the parameters concerned are linked by calculation. Considering the predictive regressions
(Table 4), sward characteristics and even herbage CP content accounted for little of OMi (Table 4), explaining only $16 \%$ of OMi variance.

OMd was well correlated with several sward characteristics with correlation coefficients in the range 0.70-0.77: leaf mass and leaf length, CP content, stem mass and sward height or extended tiller length (Table $3 a$ ). With respect to ingestive behavioural parameters, OMd was negatively correlated with biting rate and grazing time (Table $3 a$ ). It was positively correlated with bite size and intake rate but, as already pointed out for $\mathrm{OMi}$, these parameters are 


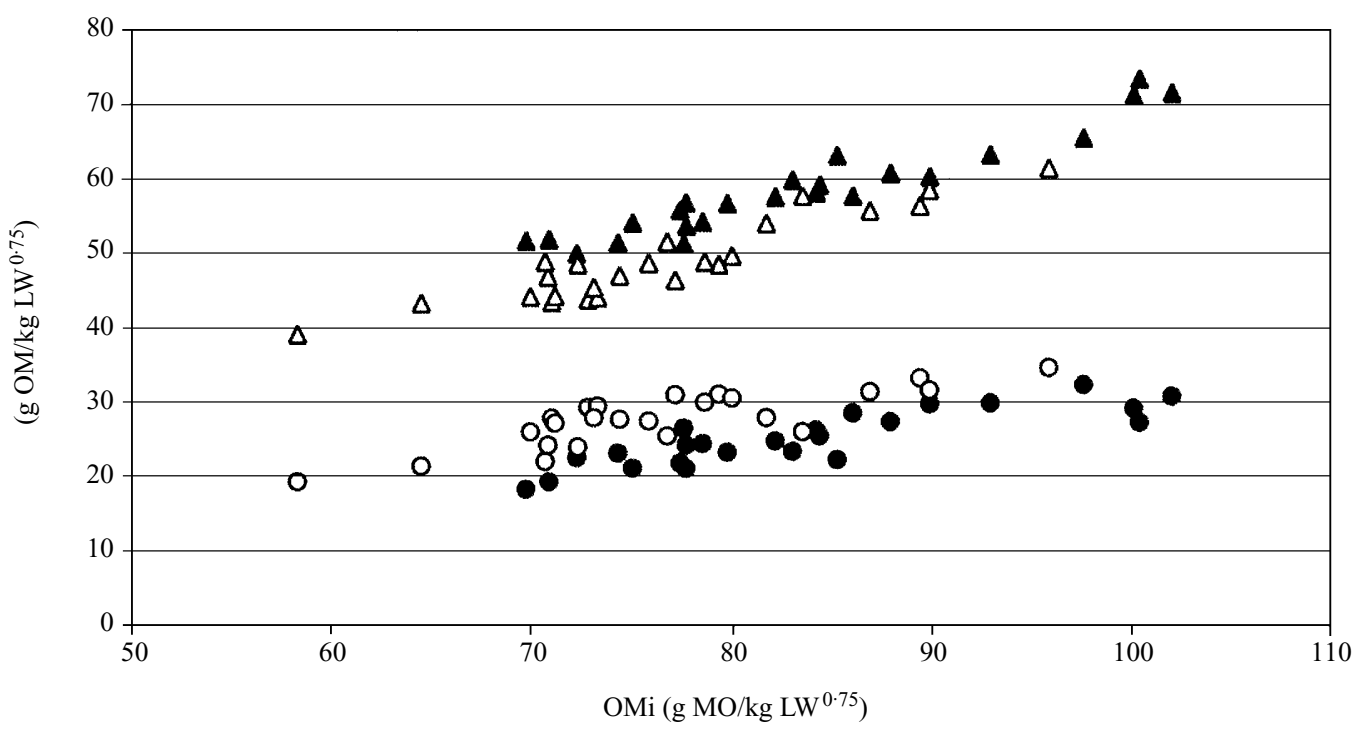

Fig. 1. Evolution of non digestible OM intake (ndOMi) and digestible OM intake (dOMi) with total OM ingested (OMi) in fertilized (F) or unfertilized (NF) Dichanthium spp. based sward. $\mathbf{\Delta}$, domi-F; $\triangle$, domi-NF; $\mathbf{O}$, ndOMi-F; $\bigcirc$, ndOMi-NF.

Table 4. Predictive regressions of $O M$ intake $\left(O M i, g / k g ~ L W^{0.75}\right), O M$ digestibility $(O M d)$, Biting rate $(B R$, bites/min), Bite size (BS, mg OM), Intake rate $(\mathrm{g} O M / \mathrm{min})$, Grazing time $(G T$, min) and Ruminating time $(R T$, min), from characteristics of tropical Dichanthium spp. based swards grazed by tethered Creole heifers

\begin{tabular}{|c|c|c|c|c|}
\hline Equations & $\begin{array}{c}\text { Percentage } \\
\text { variance } \\
\text { explained } \\
\left(R^{2}\right)\end{array}$ & $\begin{array}{c}\text { Residual } \\
\text { standard } \\
\text { deviation } \\
\text { (R.s.d) }\end{array}$ & $\begin{array}{c}\text { Error } \\
\text { degrees } \\
\text { of freedom } \\
\text { (D.F.) }\end{array}$ & Mean \\
\hline $\mathrm{OMi}=47 \cdot 11+49 \cdot 18 \mathrm{OMd}$ & $0 \cdot 02$ & $9 \cdot 43$ & 46 & \multirow[t]{4}{*}{$80 \cdot 1$} \\
\hline $\mathrm{OMi}=58 \cdot 3+2 \cdot 48$ Herbage $\mathrm{CP}$ & $0 \cdot 16$ & $8 \cdot 71$ & 46 & \\
\hline $\mathrm{OMi}=41 \cdot 11+1.48 \mathrm{ndOMi}$ & $0 \cdot 37$ & $7 \cdot 64$ & 46 & \\
\hline $\mathrm{OMi}=-2.3+1.9 \mathrm{ndOMi}+3.69 \mathrm{CP}$ & $0 \cdot 74$ & $4 \cdot 86$ & 45 & \\
\hline OMd $=0.56+0.023$ Sward height -0.0008 Sward height $^{2}$ & $0 \cdot 51$ & $0 \cdot 028$ & 45 & \multirow[t]{5}{*}{$0 \cdot 67$} \\
\hline OMd $=0.59+0.018$ Stem length -0.0006 Stem length $^{2}$ & $0 \cdot 51$ & $0 \cdot 028$ & 45 & \\
\hline OMd $=0.51+0.017$ Ext. tiller length -0.0003 Ext. tiller length ${ }^{2}$ & 0.57 & $0 \cdot 026$ & 45 & \\
\hline OMd $=0 \cdot 34+0 \cdot 070 \mathrm{Ln}$ (Leaf mass) & $0 \cdot 63$ & $0 \cdot 025$ & 46 & \\
\hline $\mathrm{OMd}=0.52+0.00037$ Leaf mass +0.012 Herbage $\mathrm{CP}$ & $0 \cdot 76$ & $0 \cdot 019$ & 45 & \\
\hline $\mathrm{BR}=60.75-1.44$ Sward height & $0 \cdot 51$ & 3.92 & 46 & \multirow[t]{2}{*}{52} \\
\hline $\mathrm{BR}=112 \cdot 6-1 \cdot 108$ Sward height -0.754 Herbage NDF & $0 \cdot 57$ & $3 \cdot 62$ & 45 & \\
\hline $\mathrm{BS}=96 \cdot 5+547 \cdot 22$ Stem fraction & $0 \cdot 58$ & $35 \cdot 1$ & 46 & \multirow[t]{4}{*}{226} \\
\hline $\mathrm{BS}=96 \cdot 51+386 \cdot 75$ Stem fraction $+6 \cdot 15$ Sward height & $0 \cdot 62$ & $33 \cdot 4$ & 45 & \\
\hline $\mathrm{BS}=-2 \cdot 35+26 \cdot 07$ Herbage CP & 0.63 & $32 \cdot 9$ & 46 & \\
\hline $\mathrm{BS}=11 \cdot 65+19 \cdot 30$ Herbage $\mathrm{CP}+7 \cdot 34$ Sward height & $0 \cdot 73$ & $28 \cdot 6$ & 45 & \\
\hline $\mathrm{IR}=6 \cdot 8+20 \cdot 19$ Stem fraction & $0 \cdot 39$ & $1 \cdot 86$ & 46 & \multirow[t]{3}{*}{$11 \cdot 6$} \\
\hline $\mathrm{IR}=1.78+1.12$ Herbage CP & 0.59 & $1 \cdot 54$ & 46 & \\
\hline $\mathrm{IR}=3.89+1.18$ Herbage $\mathrm{CP}-7.40$ Leaf fraction & $0 \cdot 61$ & $1 \cdot 48$ & 45 & \\
\hline $\mathrm{GT}=283 \cdot 4+63.9$ Debris bulk density & $0 \cdot 38$ & $51 \cdot 4$ & 46 & \multirow[t]{2}{*}{396} \\
\hline $\mathrm{GT}=485.9+7 \cdot 9$ Total bulk density $-21 \cdot 3$ Herbage CP & $0 \cdot 44$ & $48 \cdot 9$ & 45 & \\
\hline $\mathrm{RT}=355 \cdot 9-37 \cdot 34$ Total bulk density & $0 \cdot 26$ & $68 \cdot 4$ & 46 & \multirow[t]{2}{*}{154} \\
\hline $\mathrm{RT}=176 \cdot 7-28.9$ Total bulk density -451 Herbage DM & $0 \cdot 38$ & $62 \cdot 6$ & 45 & \\
\hline
\end{tabular}

Ext. tiller length, Extended tiller length. 

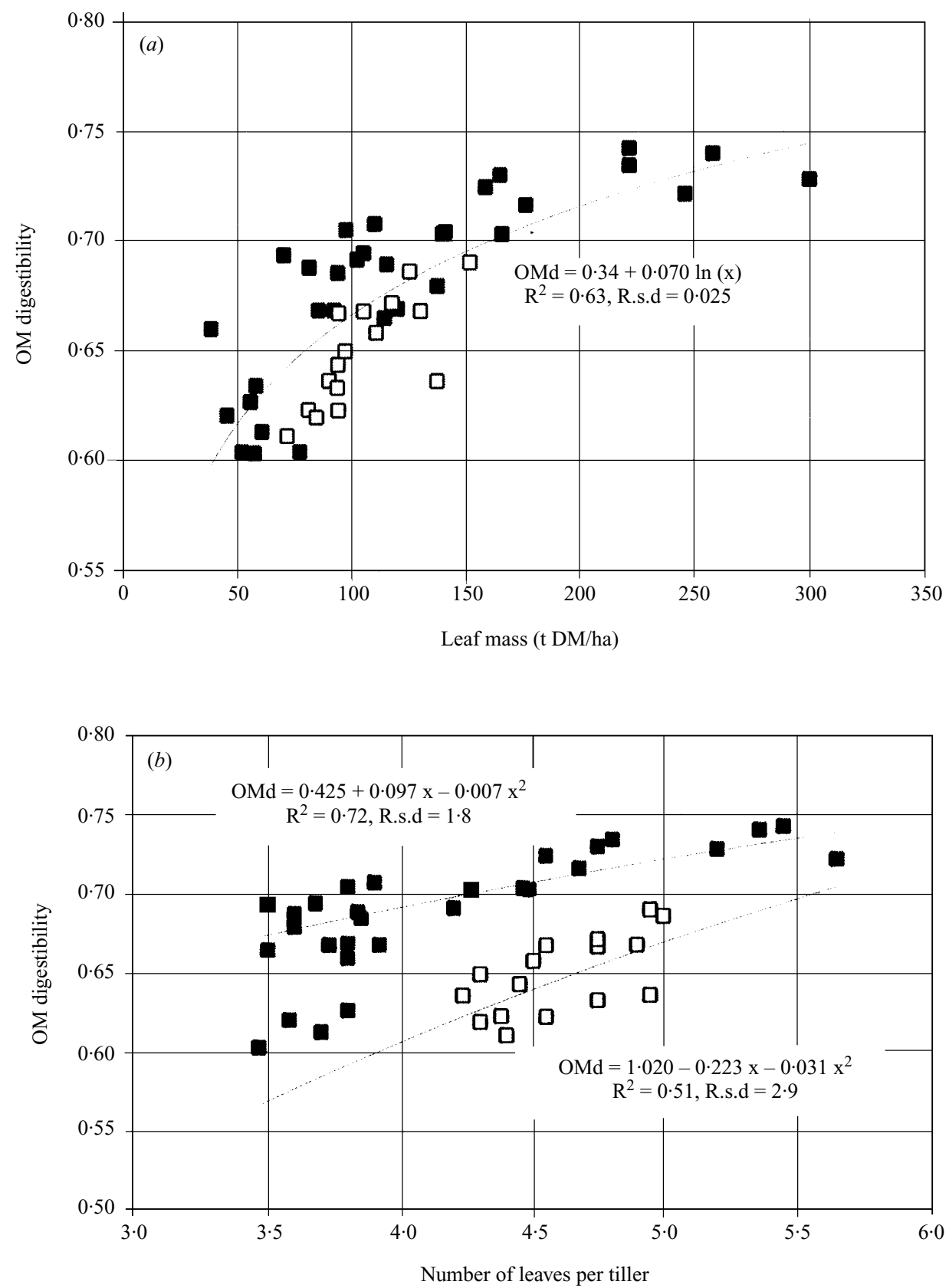

Fig. 2. (a). Effect of leaf mass on in vivo OM digestibility (OMd) measured on fertilized (F, $\square$ ) or unfertilized sward (NF, $\square$ ) Dichanthium spp. based swards. (b) Effect of the number of leaves per tiller on in vivo OM digestibility (OMd) on fertilized $(\mathrm{F}, \boldsymbol{\square})$ and unfertilized (NF, $\square)$ Dichanthium spp. based swards.

linked to OMd by calculation. The predictive regression analysis shows that leaf mass was the main determining parameter for OMd. Indeed leaf mass alone explained $63 \%$ of OMd variance (Table 4, Fig. $2 a$ ) and, combined with the CP content, $R^{2}$ went up to $76 \%$ (Table 4). Stem length and sward height predicted OMd equally and the prediction was slightly better on the basis of extended tiller length. Considering $\mathrm{F}$ sward data separately, the number of leaves appeared to be the parameter which is best 


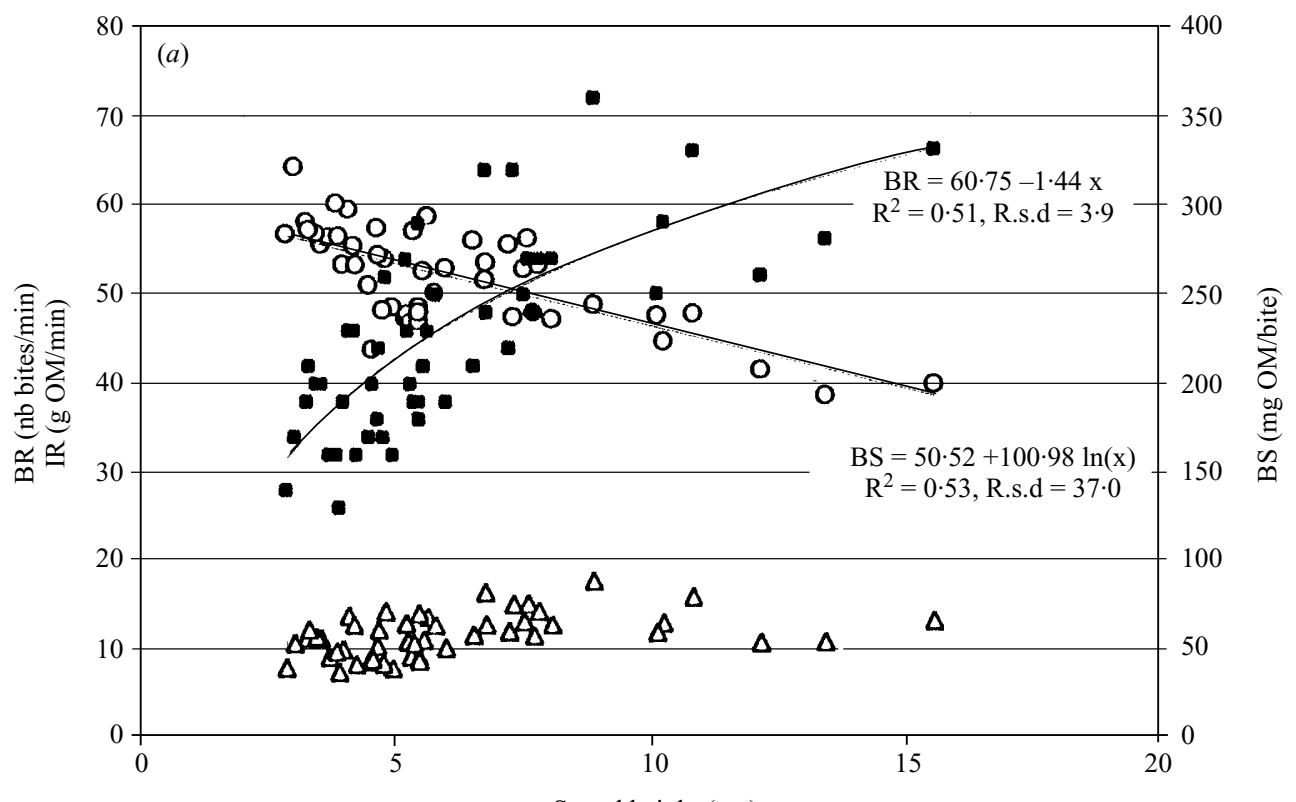

Sward height $(\mathrm{cm})$

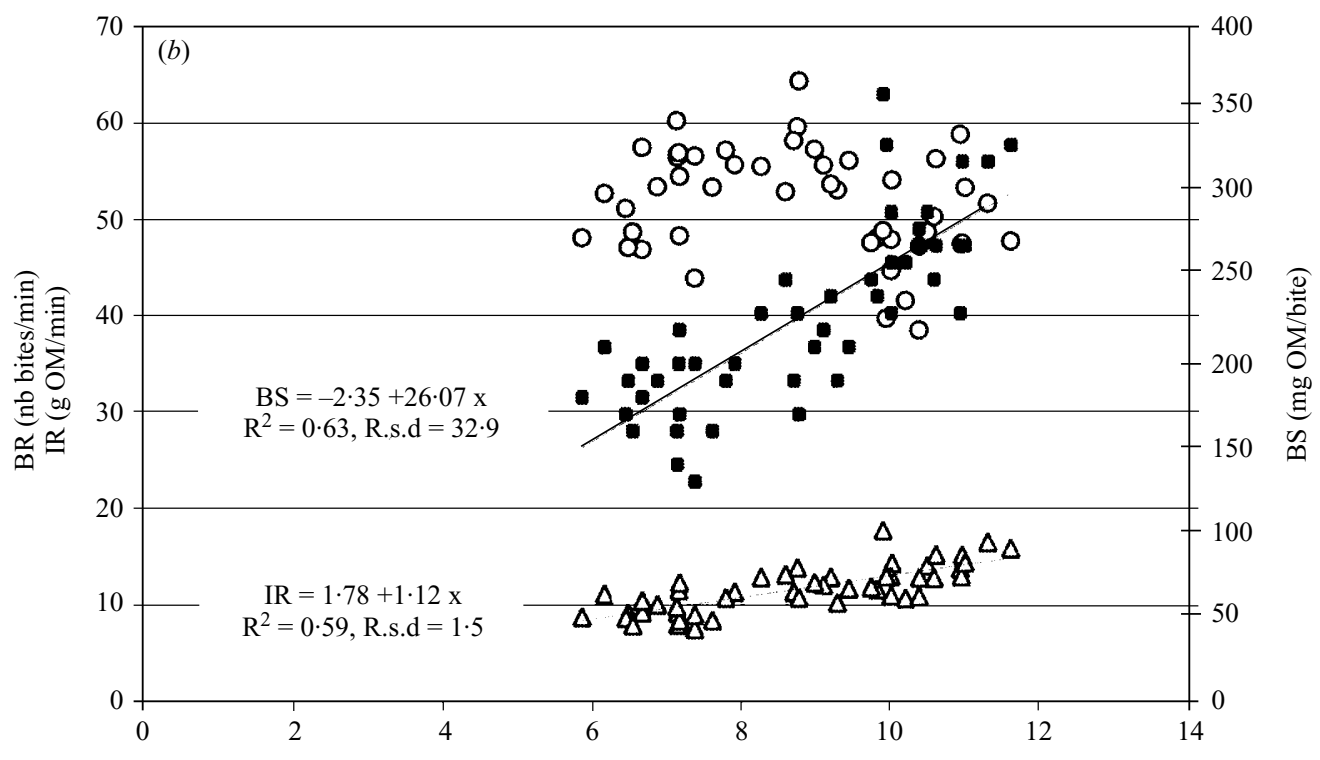

Herbage CP content (\% DM)

Fig. 3. (a). Evolution of biting rate (BR, O), intake rate (IR, $\triangle$ ) and bite size (BS, $\square$ ) with sward height of a Dichanthium spp. based sward, with or without fertilization, $(b)$ with CP content of a Dichanthium spp. based sward, with or without fertilization.

correlated with OMd (Fig. $2 b$ ). This relationship was also significant in the NF sward.

The feeding behavioural parameters such as biting rate, bite size and intake rate were better correlated with sward characteristics than with grazing, ruminating or idling times. Biting rate was well correlated with, and predicted by sward height (Tables $3 a$ and 4 , Fig. $3 a$ ). Bite size and intake rate were first correlated with CP content (Fig. $3 b$ ), and to a lesser extent with stem fraction. Grazing and ruminating times were both predicted mainly by total bulk density. 


\section{DISCUSSION}

\section{Effect of nitrogen fertilizer and successive grazing cycles on Dichanthium spp. characteristics}

The rise in herbage mass induced by the addition of nitrogen fertilizer was consistent with other reports, from $19 \mathrm{~kg}$ DM per additional $\mathrm{kg} \mathrm{N}$ for Digitaria decumbens (Ethredge et al. 1973; Blunt \& Haydock 1977) to $29.7 \mathrm{~kg} \mathrm{DM} / \mathrm{kg} \mathrm{N}$ for Cynodon dactylon (Crespo 1984), two stoloniferous tropical grasses as are also Dichanthium spp. The higher CP content due to nitrogen fertilizer has also been reported in other tropical studies (Minson 1973; Monson \& Burton 1982), whereas the rise we measured was greater, probably because of measurement conditions at pasture.

The rise in herbage mass and $\mathrm{CP}$ content under nitrogen fertilizer, is classically explained by leaf elongation and consequently a rise in leaf mass and leaf area index, for tillering grass species. These morphological modifications have been reported for temperate (Mazzanti et al. 1994) and tropical (Pinto et al. 1994) tillering grass species. In our experiment, the nitrogen effect on the Dichanthium spp. sward appeared mainly on the stems, which grew much longer than the leaves, with the leaf/stem ratio decreasing. This has previously been reported for Dichanthium aristatum (Schemoul 1988; Cruz \& Boval 2000), Digitaria decumbens (Cruz et al. 1989) and Cynodon dactylon (Overman \& Wilkinson 1989). Although to a lesser extent than the stems, the leaves also elongated and the greater leaf mass that followed helped to increase the $\mathrm{CP}$ content and the herbage quality. Moreover, it is possible that the addition of fertilizer doubled leaf density at the top of the sward compared to the lower layers, as shown by Stobbs (1973b, 1975) and Hendricksen \& Minson (1985), easing thereby the accessibility of leaves for grazing animals.

It is generally considered that stem elongation causes a fall in the quality of the herbage offered, but this undoubtedly proves to be invalid under fertilization. The young elongated stems caused by fertilizer may be similar in digestibility to the leaves (Haggar \& Ahmed 1970) and they do have less tensile strength than unfertilized stems (Flores et al. 1993). Furthermore, whereas the total herbage bulk density declined with the elongation of the stems under fertilizer, it remained higher than the threshold value of $100 \mathrm{~kg} \mathrm{DM} /$ ha per $\mathrm{cm}$, usually reported for tropical swards (Stobbs 1973a), which does not limit the bite size of grazing animals (Humphreys 1991). In such conditions the fertilized elongated stems may help grazing animals to get a good grip on high quality herbage.

Throughout the successive grazing cycles, a higher number of living leaves per plant, which were also much longer, was observed. This effect was measured on the NF sward as well on the F sward. Moreover, on the F sward all the other sward parameters varied, owing to the accumulation of nitrogen fertilizer from one application to another, combined with the effect of successive grazing cycles (Mears \& Humphreys 1974). The higher leaf number may be explained by the successive frequent defoliation which can generate the production of shorter leaves and sheaths than with infrequent cutting, according to Davies (1977).

In the current study some typical effects of nitrogen fertilization, such as the rise in herbage mass, CP content and leaf elongation, were demonstrated for a grazed Dichanthium spp. based sward. However, the fertilizer effect on stem elongation is more specific to tropical stoloniferous grasses. In other tropical studies, the leaf expansion rate measured throughout the successive grazing cycles was not reported either. In fact there is a lack of characterization of various morphological tropical grasses at pasture (Hacker \& Evans 1992; Cruz \& Boval 2000).

\section{Nitrogen fertilizer effect on intake and digestibility}

At a fixed daily herbage allowance, intake and digestibility were higher for the fertilized sward, resulting in a rise in digestible $\mathrm{OM}$ intake (dOMi) by $20 \cdot 6 \%$. Minson (1973) with tropical Chloris gayana and Delagarde et al. (1997) with temperate perennial ryegrass, reported dOMi rises of 21 and $25 \cdot 6 \%$, from fertilizing respectively with 125 to $500 \mathrm{~kg} \mathrm{~N} / \mathrm{ha}$ at 28 days of re-growth and from 0 to $60 \mathrm{~kg} \mathrm{~N} / \mathrm{ha}$ at 32 days of re-growth. The $20.6 \%$ increase in dOMi is consistent with the LW gain during the experiment, for the NF and F swards (respectively 230 and $462 \mathrm{~g} /$ day), although it was measured over short periods. The energy input is sufficient to meet maintenance requirements $\left(0.044 \mathrm{UF} / \mathrm{kg} \quad \mathrm{LW}^{0.75}\right.$, Vérité et al. 1987), and the growth energy for a potential daily LW gain of $200 \mathrm{~g}$ and $425 \mathrm{~g}$ respectively for the NF and F swards. Taking into account the amount of fertilizer added, the LW gain from the NF to the $\mathrm{F}$ sward is equivalent to $1.47 \mathrm{~kg} \mathrm{LW} / \mathrm{ha}$ per additional $\mathrm{kg}$ of fertilizer. This value is close to values reported in other studies, from 1.3 to $4.7 \mathrm{~kg} \mathrm{LW} / \mathrm{ha}$ per year and per additional $\mathrm{kg}$ of nitrogen fertilizer (Mears \& Humphreys 1974; Jones 1990). However, in those studies, the stocking rate was not adjusted with regard to the fertilizer level, and the LW gain recorded is generally explained by a rising herbage allowance.

The fertilizer effect measured seems in accordance with other studies, but the explanatory parameters may differ. Firstly, greater herbage allowance due to nitrogen fertilizer could not be invoked in the current experiment. On the other hand, the herbage $\mathrm{CP}$ rise from the NF to the $\mathrm{F}$ sward contributed to the increase in OMi. In the NF sward the herbage CP content is close to the limiting value reported for nitrogen deficiency in the rumen (Minson 1990). 
According to Vérité et al. (1987) 126 to $135 \mathrm{~g}$ of CP is required to digest $1 \mathrm{~kg}$ of digestible $\mathrm{OMi}$ (dOMi), whereas for the NF sward, the amount of $\mathrm{CP}$ was $115 \mathrm{~g}$. Consequently, under fertilization, the increase in herbage $\mathrm{CP}$ content may compensate for a $\mathrm{CP}$ deficiency, facilitating the cellulolytic activity in the rumen (Archimède et al. 1999) of the grazing heifers. However, the herbage CP rise accounts only for $16 \%$ of the OMi variance, and the rumen load could be the other main determining parameter for $\mathrm{OMi}$ in our experiment (Fig. 1). Ruminants are able to ingest a constant amount of nondigestible OM (ndOMi), irrespective of the total amount consumed and which is representative of the rumen load (Lehman 1941, quoted by Jarrige 1989). Whatever F or NF swards, ndOMi varied slightly with intake, keeping close to $26 \cdot 3 \mathrm{~g} \mathrm{OM} / \mathrm{kg} \mathrm{LW}^{0.75}$. However on the $\mathrm{F}$ sward, ndOMi represented $29 \%$ of the total intake whereas it represented $36 \%$ on the NF sward (Fig. 1). So on the F sward, the amount of ndOMi was reached when a greater amount of digestible $\mathrm{OM}$ was ingested (dOMi) and consequently for a greater total intake, compared to the unfertilized sward. Such ndOMi values, have already been measured in previous experiments (Boval et al. 1996 b; Boval et al. 2000) and in other tropical environments, as reviewed by Kennedy (1995) for cattle and buffaloes. This needs to be checked in further experiments, in which rumen load is measured.

Intake was influenced by neither of the physical sward characteristics, whereas the latter influenced the ingestive behaviour of the heifers. Indeed sward height determines biting rate and bite size as already reported for cattle (Laca et al. 1992) and sheep (Black \& Kenney 1984; Burlinson et al. 1991). Stem fraction and herbage $\mathrm{CP}$ content also influenced bite size and intake rate. However, the other behavioural parameters, grazing and ruminating times for instance, were modified to a lesser extent by sward characteristics, except a little by herbage bulk density. It is possible that sward characteristics affect ingestive behaviour instantaneously, in the short-term, via parameters such as biting rate, bite size or intake rate. However on a daily basis there is compensation (Allden \& Whittaker 1970; Jamieson \& Hodgson 1979) between short-term parameters and this compensation may contribute to the maintenance of equal daily grazing and ruminating times. Indeed the nonlimiting amount of fresh herbage allowed daily, may have helped the heifers to adapt their behaviour to satisfy their appetite and energy requirements. This probably also explains why OMi, resulting from many bites during the day, is not affected by sward characteristics such as short-term ingestive behavioural parameters. Thereafter, if the addition of nitrogen fertilizer influences both $\mathrm{OMi}$ and ingestive behavioural parameters, it does not occur in the same way and this implies the choice of the appropriate parameters to understand grazing animal nutrition on a daily basis.

Conversely, the increase in OM digestibility in the F sward is mainly explainable by physical sward structure. Leaf mass accounts for the best OMd gain which is better still when combined with CP content. Sward characteristics such as sward height and extended tiller length also explain the OMd rise under fertilizer quite well. Thus, even if nitrogen fertilizer elongated the stems more than the leaves, the stems produced did not constitute a limit for gripping high quality herbage, as is often reported for tropical herbage (Stobbs $1973 b$; Hendricksen \& Minson 1980). There are a few references to the effect of sward characteristics on $\mathrm{OMd}$, which was mostly estimated in vitro. However, Forbes \& Coleman (1993) reported a significant effect of the leaf fraction of warm-season Bothrichloa spp. on OMd. Irrespective of leaf mass and CP content effects, the number of living leaves also determined $\mathrm{OMd}$, inducing a 5 unit gain (Fig. $2 b$ ), whatever the fertilizer level on the sward. This 5 unit gain is close to the 6 unit gain measured as the fertilizer effect. This was unexpected and suggests that for the same leaf mass, shorter but more numerous leaves may be better than a few long leaves in improving the digestibility of the herbage consumed at pasture. Indeed Duru et al. (1999) reported that shorter leaves are more digestible than longer ones, because the time required for elongation is shorter and the leaves are less mature. It is worth noting that $\mathrm{OMd}$ as well as ingestive behavioural parameters are influenced by sward characteristics, whereas OMi is not. The relationship between sward characteristics, some ingestive behavioural parameters and $\mathrm{OMd}$, can be explained by the fact that low quality individual bites have a higher influence on OMd than on OMi. OMi is indeed the resultant of many small amounts of herbage consumed per bite, throughout the day, and a given daily OMi can be reached by increasing either bite size, biting rate or grazing time (Allden \& Whittaker 1970; Hodgson 1982). In return, if many low quality bites are achieved by grazing animals, this induces an irremediable drop in $\mathrm{OMd}$, which is difficult to compensate.

\section{CONCLUSIONS}

Nitrogen fertilizer induced a rise in herbage mass, by way of a considerable stem and leaf elongation and a rise in herbage $\mathrm{CP}$ content. Having equal herbage allowances the heifers ingest $9 \%$ more OM, which is more digestible by 5 digestibility units, on the fertilized sward. The rise in OMi is just partly explainable by the rise in herbage $\mathrm{CP}$ content. In other respects, the stability of ndOMi regardless of nitrogen level, suggests a determining effect of rumen load on $\mathrm{OMi}$, although the latter was not measured. In contrast to $\mathrm{OMi}$, the gain in $\mathrm{OMd}$ is firstly 
explainable by physical sward characteristics such as leaf mass. The length of the fertilized stems and the subsequent sward height also help to increase OMd, probably by making the leaves easier to grip. Ingestive behaviour is closer to OMd than OMi. This suggests that studies at pasture which take into account just ingestive behaviour to indicate intake are not reliable.

Thus nitrogen fertilizer causes a significant improvement in digestible intake at pasture, by inducing a leafy sward with a greater young stem mass and a greater herbage $\mathrm{CP}$ content, irrespective of herbage allowance. This indicates that the structure of the sward has to be considered with the chemical components, to investigate ruminant nutrition at pasture. Knowledge of the effects of nitrogen fertilizer on animal nutrition via the morphological modifications of the herbage, allows better definition of suitable management for promoting nutrition at pasture. In that sense, knowledge provided by agronomists on morphological responses of tropical grass under various conditions, may be helpful.

We thank A. Nepaut and P. Mulciba for their great technical participation.

\section{REFERENCES}

Allden, W. G. \& Whittaker, I. A. McD. (1970). The determinants of herbage intake by grazing sheep: the interrelationships of factors influencing herbage intake and availability. Australian Journal of Agricultural Research 21, 755-766.

Archimede, H., Aumont, G., Saminadin, G., Depres, E., Despois, P. \& XANDE, A. (1999). Effects of urea and saccharose on intake and digestion of a Digitaria decumbens hay by black belly sheep. Animal Science $\mathbf{6 9}$, 403-410.

Black, J. L. \& Kenney, P. A. (1984). Factors affecting diet selection by sheep. Grass and Forage Science 35, 565-578.

Blunt, C. G. \& Haydock, K. P. (1978). Effect of irrigation, nitrogen and defoliation on Pangola grass in the dry season at the Ord Valley, north-western Australia. Australian Journal of Experimental Agriculture and Animal Husbandry 18, 825-833.

Boval, M., Peyraud, J. L., Xande, A., Aumont, G., Copry, O. \& SAminadin, G. (1996a). Evaluation of faecal indicators to predict digestibility and voluntary intake of Dichanthium spp. by cattle. Annales de Zootechnie 45, 121-134.

Boval, M., Peyraud, J. L. \& Xande, A. (1996b). Effect of nocturnal enclosing and splitting offered area on herbage intake of tethered creole heifers. Annales de Zootechnie 45, 219-231.

Boval, M., Cruz, P., Peyraud, J. L. \& Penning, P. (2000). Herbage allowance effect on intake of Creole heifers tethered at pasture. Grass and Forage Science 55, 201-208.

Burlison, A. J., Hodgson, J. \& Illius, A. W. (1991). Sward canopy structure and the bite dimensions and bite weight of grazing sheep. Grass and Forage Science 46, 29-38.

Crespo, G. (1984). Variation in the response of tropical pastures to nitrogenous fertilizers througout the year. 1 . Pangola grass (Digitaria decumbens Stent) without irrigation. Cuban Journal of Agricultural Science 18, 63-73.

Cruz, P. \& Boval, M. (2000). Effect of nitrogen on some morphogenetical traits of temperate and tropical perennial forage grasses. In Grassland Ecophysiology and Grazing Ecology (Eds G. Lemaire, J. Hodgson, A. de Moraes, C. Nabinger \& P. C. de F. Carvalho), pp. 151-167. Wallingford: CAB.

Cruz, P., Alexandre, G. \& Baudot, H. (1989). Cinétique de croissance foliaire et stolonifère d'un peuplement de Digitaria decumbens au cour de la repousse. In Proceedings of the XVI International Grassland Congress,
Volume I. (Ed. Desroche), pp. 499-500. Nice: The French Grassland Society.

DAvies, A. (1977) Structure of the grass sward. In Proceedings of the International Meeting on Animal Production, from Temperate Grasslands (Dublin, Ireland), pp 36-44.

Delagarde, R., Peyraud, J. L. \& Delaby, L. (1997). The effect of nitrogen fertilization level and protein supplementation on herbage intake, feeding behaviour and digestion in grazing dairy cows. Animal Feed Science and Technology 66, 165-180.

Duru, M., Ducrocq, H. \& Feuillerac, E. (1999). In vitro digestibility response of cocksfoot (Dactylis glomerata L.) to growth and defoliation: a simple model. Journal of Agricultural Science, Cambridge 133, 379-388.

Ethredge, J., Beaty, E. R. \& Lawrence, R. M. (1973). Effects of clipping height, clipping frequency, and rates of nitrogen on yield and energy content of Coastal Bermudagrass. Agronomy Journal 65, 717-719.

Flores, E. R., Laca, E. A., Griggs, T. C. \& Demment, M. W. (1993). Sward height and vertical morphological differentiation determine cattle bite dimensions. Agronomy Journal 85, 527-532.

Forbes, T. D. A. \& Coleman, S. W. (1993). Forage intake and ingestive behaviour of cattle grazing Old World Bluestems. Agronomy Journal 85, 808-816.

Hacker, J. B. \& Evans, T. R. (1992). An evaluation of the production potential of six tropical grasses under grazing. 1. Yield and yield components, growth rates and phenology. Australian Journal of Experimental Agriculture 32, 19-27.

HagGar, R. J. \& Ahmed, M. B. (1970). Seasonal production of Andropogon gayanus. 2. Seasonal changes in digestibility and feed intake. Journal of Agricultural Science, Cambridge 75, 369-373.

Hendricksen, R. E. \& Minson, D. J. (1985). The voluntary intake, digestibility and retention time by cattle and sheep of stem and leaf fractions of a tropical legume (Lablab purpureum). Australian Journal of Experimental Agriculture 32, 389-398.

Hendricksen, R. E. \& Minson, D. J. (1980). The feed intake and grazing behaviour of cattle grazing a crop of Lablab purpureus cv. Rongai. Journal of Agricultural Science, Cambridge 95, 547-554.

Hodgson, J. (1982). Influence of sward characteristics on diet selection and herbage intake by the grazing animal. In 
Nutritional Limits to Animal Production from Pastures (Ed. J. B. Hacker), pp. 153-166. Farnham Royal: Commonwealth Agricultural Bureaux.

Humphreys, L. R. (1991). Tropical Pasture Utilisation. Cambridge: Cambridge University Press.

Jamieson, W. S. \& Hodgson, J. (1979). The effect of daily herbage allowance and sward characteristics upon the ingestive behaviour and herbage intake of calves under strip-grazing management. Grass and Forage Science 34, 261-271.

JARRIGE, R. (1989). Recommended Allowances and Feed Tables (Ed. R. Jarrige). Paris: INRA.

JONES, R. J. (1990). Nitrogen rate and stocking rate effects on steer gains from grazed irrigated pangola grass in the Ord Valley, Western Australia. Australian Journal of Experimental Agriculture 30, 599-605.

KenNedy, P. M. (1995) Comparative adaptability of herbivores to tropical environments. In Proceedings of the IVth Symposium on the Nutrition of Herbivores (Eds M. Journet, E. Grenet, M.-H. Farce, M. Theriez \& C. Demarquilly), pp. 309-328. Paris: INRA.

Laca, E. A., Ungar, E. D., Seligman, N. G. \& Demment, M. W. (1992). Effects of sward height and bulk density on bite dimensions of cattle grazing homogeneous swards. Grass and Forage Science 47, 91-102.

Mazzanti, A., Lemaire, G. \& Gastal, F. (1994). The effect of nitrogen fertilization upon the herbage production of tall fescue swards continuously grazed with sheep. 1. Herbage growth dynamics. Grass and Forage Science 49, $111-120$.

Mears, P. T. \& Humphreys, L. R. (1974). Nitrogen response and stocking rate of Pennisetum clandestinum pastures. 2. Cattle growth. Journal of Agricultural Science, Cambridge 83, 469-478.

MicheLl, P. (1982). Value of rising-plate meter for estimating herbage mass of grazed perennial ryegrass-white clover swards. Grass and Forage Science 37, 81-87.

Minson, D. J. (1973). Effect of fertiliser nitrogen on digestibility and voluntary intake of Chloris gayana digitaria decumbens and Pennisetum clandestinum. Australian Journal of Experimental Agriculture and Animal Husbandry 13, 153-157.

Minson, D. J. (1990) In Forage in Ruminant Nutrition (Ed. D. J. Minson). San Diego: Academic Press.
Monson, W. G. \& Burton, G. W. (1982). Harvest frequency and fertilizer effects on yield, quality, and persistence of eight Bermudagrasses. Agronomy Journal 74, 371-374.

Overman, A. R. \& Wilkinson, S. R. (1989). Partitioning of dry matter between leaf and stem in Coastal Bermudagrass. Agricultural Systems 30, 35-47.

Pinto, J. C., Gomide, J. A., Maestri, M. \& Lopes, N. F. (1994). Crescimento de folhas de gramineas forrageiras tropicales, cultivadas en vasos, com duas doses de nitrogenio. Revista da Sociedade Brasileire de Zooteccnia 23, 327-332.

SAS (1989) SAS/STAT User's guide, Version 6, Fourth Edition, volume 2. Cary, NC: SAS Institute Inc.

Schemoul, E. (1988). Productivité primaire et morphogénèse d'un peuplement de Petit Foin (Dichanthium spp. Will) en Guadeloupe. Mémoire DAA, ENSA Montpellier.

Stoввs, T. H. (1973a). The effect of plant structure on the intake of tropical pastures. I. Variation in the bite size of grazing cattle. Australian Journal of Agricultural Research 24, 809-819.

Stoвbs, T. H. (1973b). The effect of plant structure on the intake of tropical pastures. II. Difference in sward structure, nutritive value, and bite size of animals grazing Setaria anceps and Chloris gayana at various stages of growth. Australian Journal of Agricultural Research 24, 821-829.

Stoввs, T. H. (1975). The effect of plant structure on the intake of tropical pastures. 3. Influence of fertiliser nitrogen on the bite size harvested by Jersey cows grazing Setaria anceps CV. Kazungula swards. Australian Journal of Agricultural Research 26, 997-1007.

STREeTER, C. L. (1969). A review of techniques used to estimate in vivo digestibility of grazed forage. Journal of Animal Science 29, 757-768.

Tilley, J. M. A. \& Terry, R. A. (1963). A two stages technique for in vitro digestion of forage crops. Journal of the British Grassland Society 18, 104-111.

Van Soest, P. J., Robertson, J. B. \& Lewis, B. A. (1991). Methods for dietary fibre, neutral detergent fibre and non starch polysaccharides in relation to animal nutrition. Journal of Dairy Science 74, 3583-3597.

Vérité, R., Michalet-Doreau, B., Chapoutot, P., Peyraud, J. L. \& Poncet, C. (1987). Révision du système des protéines digestibles dans l'intestin (P.D.I). Bulletin Technique du C.R.Z.V. de Theix, I.N.R.A 70, 19-34. 